\title{
RAMAN SPECTROSCOPY OF SOLAR P-MODES
}

\author{
M.P. RYUTOVA AND T.D. TARBELL \\ Stanford-Lockheed Institute for Space Research, LMATC, \\ 3251 Hanover Street, Palo-Alto, CA 94304, USA
}

\begin{abstract}
According to observational data some regions of quiet sun show an excess, or the "emission" of acoustic waves. We propose a mechanism to explain the excess of the emission of acoustic waves based on nonlinear coupling of magnetic flux tube oscillations and the acoustic waves. We also suggest a new approach in the data analysis, which can be called "Raman spectroscopy of p-modes". This new idea is based on the fact that oscillating flux tubes constructively interfere with the acoustic wave which results in the generation of beat waves with combinational frequencies: the power spectra of scattered waves in addition to main peak will have Stokes and anti-Stokes satellites. Their amplitude and frequency shift reflect the properties and the structure of the observed region.
\end{abstract}

\section{Introduction}

The presence of the constant level of the solar p-mode fluctuations is a reflection of the dynamic balance between two processes: generation of p-modes by turbulent motions, and their damping by classical and/or anomalous dissipation mechanisms. Studies of the acoustic wave properties show that the sources and sinks are distributed nonuniformly over the solar surface, with sinks associated predominantly with sunspot and plage regions (Braun 1995; LaBonte \& Ryutova 1993). The sources are more likely connected with the turbulent motions outside magnetic regions and, according to Brown's conjecture (1991), may be isolated and well separated from one another in space and time. Localized sources of the acoustic waves (Brown et al. 1992) and excess of the acoustic emission (Bogdan et al. 1993; Braun 1995) were observed in the quiet sun regions with the enhanced network of small scale magnetic elements. We propose a physical mechanism to explain the excess of the emission in the quiet Sun based on the interaction of p-modes with non-steady state motions, and in particular with the oscillating magnetic flux tubes. Note, that only non-steady motions can give rise to the increase of the energy of outgoing waves. We find two major effects that may contribute to this process. One is a resonance scattering: the energy of p-modes propagating in the random ensembles of flux tubes damps out due to the 


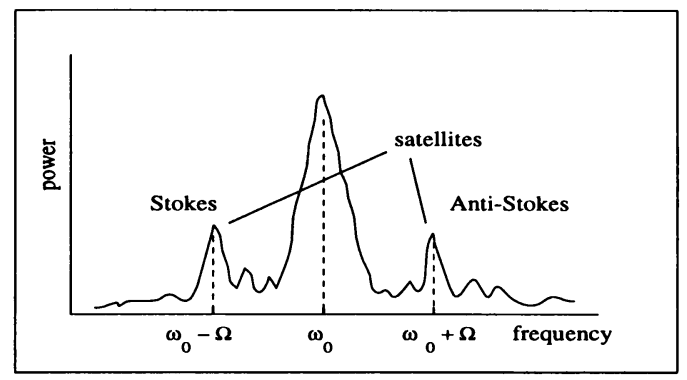

Figure 1. The sketch of power spectrum in Raman scattering.

mechanism similar to Landau damping, which consists in resonance excitation of natural oscillations of magnetic flux tubes (Ryutov \& Ryutova 1976; Ryutova \& Priest $1993 \mathrm{a}, \mathrm{b})$. The transformed energy of p-modes remains for a "long time" in a form of flux tube oscillations. Then, in a time $\tau_{\text {rad }} \simeq 1 / \omega(k R)^{2}$, the resonant flux tubes radiate accumulated energy into surrounding plasma ( $R$ is a typical flux tube radius). Because $\tau_{\text {rad }}$ depends on flux tube radius, different flux tubes radiate secondary acoustic waves in different times, which is consistent with the conjecture by Brown. The resonance (Cherenkov) condition is determined by sound speed and parameters of flux tube: $\omega=(\mathbf{k} \cdot \mathbf{n}) v_{p h}$, where $\mathbf{n}$ is a unit vector along the tube axis, and $v_{p h}$ is the phase speed of excited tube oscillation, either kink $(m= \pm 1)$ or sausage $(m=0)$ mode. Depending on the actual distribution of magnetic elements the power of outgoing waves may be considerably higher than incoming wave power. However, the radiation may occur only if the phase velocity of flux tube oscillations exceeds the sound speed in the ambient plasma: $v_{p h}>c_{s e}$. Another effect works in the opposite case when $v_{p h}<$ $c_{s}$ and is connected with nonlinear coupling of flux tubes oscillations and acoustic waves. This nonlinear coupling leads to appearance of beat waves with combinational frequencies. The power spectrum of outgoing wave will have then along the peak at the main frequency, the Stokes and anti-Stokes satellites that reflect the properties and structure of the observed medium.

\section{Raman scattering of p-modes.}

Oscillations of flux tubes may be excited by the convective motions or by fast reconnection processes. We consider the situation when oscillations of flux tubes are excited to a non-negligible level and have non-steady character. Then, nonlinear terms in the MHD equations cause a coupling of the incoming acoustic wave, and oscillations of flux tube. Which, in turn, leads to generation of beat waves with combinational frequency, $\omega_{i n} \pm \Omega$ and wave number $k_{i z} \pm K_{z}$ (Figure 1). The frequency shift, $\Omega$ does not correspond to any of the eigen-frequencies of the p-modes; this is a perturbation characteristic to each particular region, and may correspond, for example to eigenfrequency of a "scattering center" (see below). The effective distance at which waves interact with given flux tube is of the order of flux tube radius, $R$. Main component of pressure perturbation in the vicinity of the magnetic flux is axisymmetric: $\delta p \simeq k \xi_{a c} p ; \delta v \simeq \omega \xi_{a c} k R ; \delta R / R \simeq \delta p / p$. This is coupled with $m= \pm 1$ flux tube motions. The resulting nonlinear drive exists at the scale $\simeq R$, has $m= \pm 1$ symmetry, and can be expressed as the following "equivalent" displacement $\delta x \simeq \xi_{k i n k} k \xi_{a c}$. $\delta x$ 
can be conceived as a "forcing" term for generating the secondary emission. Assuming that $\omega=\omega_{\text {in }} \simeq \Omega, k_{z}^{i n} \simeq K_{z}$, one can find an estimate for the power radiated per unit length of flux tube, $P_{\text {Raman }}$. If the density of flux tubes (number of flux tubes in the square of a unit area) is $\sigma$, then the power scattered by the volume of the size $L \times L^{2}$, is $\sigma P_{\text {Raman }} L^{3}$. Incident acoustic energy flow into the same volume is $\xi_{a c}^{2} k^{2} \rho c_{s e}^{3} L^{2}$. The ratio of the two is a measure of the excess of the emitted acoustic power and has a form: $\kappa=\sigma L(k R)^{4} k \xi_{k i n k}^{2}$. Obviously, quantity $\kappa$ has a meaning of the "optical depth" with respect to the scattering process. For the sausage oscillations, $\kappa=\sigma L(k R)^{2} k \xi_{\text {saus }}^{2}$. Using the amplitude of satellites an frequency shift one can infer physical parameters of "scattering centers", and develop the analysis to study the structure of atmosphere through the spectroscopic features of p-modes.

\section{Examples and Discussion}

We give some examples of measured power spectra of incoming and outgoing waves and their possible interpretation, only to demonstrate the principle of the method. The power spectra was obtained by Doug Braun using the Hankel decomposition method for MDI/SOHO high resolution data set. The target center was a very quiet region. The power spectra of incoming and outgoing waves for four values of spherical degree, $\ell$, are given in Table $1 . P_{\text {in }}$ and $P_{\text {out }}$ are values (in arbitrary units) of net power averaged over a narrow frequency interval $(0.23 \mathrm{mHz})$ near the peaks in outgoing power spectrum. We use first three cases, $\ell=452.6 ; 699.4$ and 740.6 , as an il-

TABLE 1. Power spectra and estimates for inferred Alfvén speed.

\begin{tabular}{|c|c|c|c|c|c|c|}
\hline$\ell$ & $P_{i n}$ & $P_{\text {out }}$ & $\nu(m H z)$ & $\nu_{0}-\nu_{\mp}$ & $c_{s} k m s^{-1}$ & $v_{A} k m s^{-1}$ \\
\hline \multirow{3}{*}{452.6} & $2.1410^{3}$ & $1.4410^{4}$ & $\nu_{-}=2.67$ & 0.55 & \multirow{3}{*}{31.1} & \multirow{3}{*}{5.30} \\
\hline & $3.4610^{4}$ & $2.7210^{4}$ & $\nu_{0}=3.22$ & & & \\
\hline & $1.3110^{4}$ & $3.1010^{4}$ & $\nu_{+}=3.77$ & -0.55 & & \\
\hline \multirow{3}{*}{699.4} & $4.9610^{3}$ & $9.9010^{3}$ & $\nu_{-}=2.60$ & 0.60 & \multirow{3}{*}{20.0} & \multirow{3}{*}{3.75} \\
\hline & $3.5210^{4}$ & $3.5110^{4}$ & $\nu_{0}=3.20$ & & & \\
\hline & $2.7510^{4}$ & $4.4810^{4}$ & $\nu_{+}=3.8$ & -0.60 & & \\
\hline \multirow{3}{*}{740.6} & $1.5410^{4}$ & $1.6910^{4}$ & $\nu_{-}=2.70$ & 0.62 & \multirow{3}{*}{19.6} & \multirow{3}{*}{3.66} \\
\hline & $2.3210^{4}$ & $5.9010^{4}$ & $\nu_{0}=3.32$ & & & \\
\hline & $2.7810^{4}$ & $3.2510^{4}$ & $\nu_{+}=3.94$ & -0.62 & & \\
\hline \multirow{3}{*}{905.1} & $1.3510^{4}$ & $5.3710^{3}$ & $\nu_{-}=3.06$ & 0.55 & \multirow{3}{*}{17.4} & \multirow{3}{*}{$\mathrm{N} / \mathrm{A}$} \\
\hline & $2.9310^{4}$ & $5.7610^{4}$ & $\nu_{0}=3.61$ & & & \\
\hline & $1.3110^{4}$ & $3.1010^{4}$ & $\nu_{+}=3.77$ & -0.69 & & \\
\hline
\end{tabular}

lustration of possible Raman scattering, and the fourth case, $\ell=905.1$ as an example of resonance emission. In the first three cases the satellite peaks in outgoing power spectra exceed their counterparts in the incoming power spectra and are shifted equally from the central peak. The "Stokes" and "anti-Stokes" shifts may be used to infer the characteristic Alfvén speed. Indeed, in the subsurface layers Alfvén velocity is small 
compared to sound speed, therefore the phase velocities of both, kink and sausage mode of flux tube oscillations is close to the Alfvén speed, i.e. $v_{p h}=\Omega / k \simeq v_{A}$, where $\Omega=2 \pi\left|\nu_{0}-\nu_{ \pm}\right|$. Assuming that the wave number is approximately the same as that of incident acoustic wave we can express it through sound speed and write an estimate as: $v_{A} \simeq c_{s} \Omega / \omega_{i n}$. For given spherical degree $\ell$ and frequency $\nu$ the local sound speed is roughly estimated as $c_{s}(l, \nu)=2 \pi R_{\odot} \nu / l$. Results of the inferred Alfvén speed are given in the last columns of the table. For $\ell=905.1$ this estimate is not applicable: there are no equidistant satellites. But one can see that the outgoing wave power is considerably higher than the incoming wave power which may be provided by reemission of previously accumulated energy by resonant flux tubes.

The future observations and data analysis should be directed toward detailed study of high resolution magnetograms and wave field associated with compact isolated regions. One should bear in mind some distinct specific features of both possible effects, resonance emission and Raman scattering. For example, in a case of resonance absorption and radiation of secondary acoustic waves the necessary condition for the accumulation of acoustic wave power in the oscillating flux tubes is that the radiative damping time must be larger than the inverse Landau damping rate; this implies the condition that the acoustic wavelength must be larger than the average distance between the flux tubes: $\lambda \gg d$. Which means that the regions with high population of small scale structures will contribute to resonance scattering most efficiently. The intensity of main peak in outgoing waves may considerably exceed the main peak of incoming power. In the case of Raman scattering the most prominent feature is the appearance of equidistant Stokes and anti-Stokes satellites in the power spectrum of outgoing waves. The emitted power is proportional to magnetic filling factor and size of the observed area. It is more sensitive to small parameter $(k R)^{2}$ than the power radiated by resonant flux tubes. On the other hand, in contrast to the resonance case where the condition $(k R)^{2} \ll 1$ is required, here the parameter $(k R)^{2}$ may be finite. Therefore, large regions containing flux tubes comparable with the acoustic wave length, will contribute the Raman scattering most readily. Good candidates here may be the quiet photospheric network near plages and regions containing small pores.

\section{Acknowledgments}

We thank Doug Braun for providing the data and for helpful comments. This research is supported by NASA MDI contract NAG5-3077 ( PR 9162) at Lockheed.

\section{References}

Bogdan,T, Brown, T.M., B.W. Lites \& J.H. Thomas, (1993) The Absorption of p-modes by Sunspots, $A p J$, Vol. 406, pp. 723-734

Braun, D.C., (1995) Scattering of p-Modes by Sunspots. I. Observations, $A p J$, Vol. 451, pp. $859-876$

Brown, T.M. (1991) The Source of Solar High-Frequency Acoustic Modes: Theoretical Expectations, $A p J$, Vol. 371, pp. 396-401

Brown, T.M., T. Bogdan, B. Lites and J. Thomas (1992) Localized Sources of Propagation Acoustic Waves in the Solar Photosphere, ApJ, Vol. 394, pp. L65-L68

LaBonte, B. \& Ryutova, M. (1993) A Possible Mechanism for Enhanced Absorption of pModes in Sunspot and Plage Regions, $A p J$, Vol. 419, pp. 388-397

Ryutov, D.D.\& Ryutova, M.P. (1976) Sound oscillations in a plasma with magnetic filaments, Sov.Phys. JETP, Vol. 43, pp. 491-497

Ryutova, M.P. \& Priest, E.R. (1993a;b) The Propagation of Sound Waves in a Randomly Magnetized Medium. ApJ, Vol. 419, pp. 349-370; pp. 371-381 